\title{
Structural flexibility and disorder in functional materials
}

\section{Andrew Goodwin}

\author{
University of Oxford, Oxford, United Kingdom; \\ andrew.goodwin@chem.ox.ac.uk
}

Flexible materials can adapt their structures easily in response to external stimuli. For this reason, they are often used as sensors or actuators; they can show useful and unusual mechanical behaviour such as negative thermal expansion or negative compressibility. In a conceptually related manner, disordered materials navigate a shallow configurational landscape of degenerate states. This degeneracy also often heightens their susceptibility to external perturbations. This lecture will explore the fundamental design principles associated with structural flexibility and correlated disorder, and their role in a range of functional materials. Case studies taken from our own work will includie metal-organic frameworks, oxide ceramics, and frustrated magnets.

Keywords: correlated disorder, metal-organic frameworks, diffuse scattering, flexibility 